\title{
ECUADOR MAMALLAKTAPAK MAMAKAMACHIYTA (2008) KUTIN YUYARINA
}

\author{
REPENSANDO LA CONSTITUCIÓN ECUATORIANA DE 2008
}

RETHINKING THE ECUADORIAN CONSTITUTION OF 2008

\section{UCHILLACHISKA YUYAY}

Kay killkaka, Ecuador Mamallaktapak shuk waranka iskunpatsak, iskun chunka pusak watapi (1998) killkachishkata, shinallatak ishkay waranka pusak watapi (2008) killkachishka Mamakamachiykunatami chimpapurashpa rikuchin, tawka kawsay, tawka yuyay chikan chikan kawsaypura yuyayta rikushpa, kay yuyaykunaka runakunapak mutsurishkakunata, hatun tantankuykuna, mañaykunata rurankapak hillaykunami kan. Katipika, mama llaktapak kamay imashina kamachinakuyta paktachishmanata, shinallatak tawkapura mama llaktapi sumkata wankurishpa kawsankapak kunashkakunapashmi kan.

Sapi shimikuna: Maman llaktapak kamachiy, tawka kawsay, shukshinalla kawsay, tawkakawsayuk, kawsaypura, kamachiymanta tapuykuna.

\section{RESUMEN}

El texto analiza (a partir de las entrevistas, de la revisión bibliografica especializada y secundaria y demás materiales referentes al tema) la transición de la Constitución Política de la República del Ecuador de 1998 a la del 2008; haciendo reflexiones comparativas en el pasaje de una Constitución pluricultural y multiétnica hacia una intercultural $y$ plurinacional. Este pasaje implica el cambio de tipología de sociedad ecuatoriana, al menos en teoría, y la manera cómo estas categorías y postulados se constituyen en una caja de herramienta para las insurgencias y diálogos de los pueblos $y$ nacionalidades indigenas frente a la estructura dominante. Seguidamente, aborda los avances, limites y contradicciones de las prácticas y discursos constitucionales. Ambos conceptos: tanto la interculturalidad como la plurinacionalidad parecen apuntalarse como una contrahegemonía ontológica, epistémica y política. Epistémicamente, por ejemplo, resurgen o aparecen como saberes locales, aunque estos saberes se vuelven ensimismados; es decir, que son parte solamente de la población subalternizada, más allá de las incorporaciones que se hacen a los mismos en las academias, pero que son en su mayoría marginales.

Palabras claves: Constitución, pluricultural, multiétnico, plurinacional, referéndum.

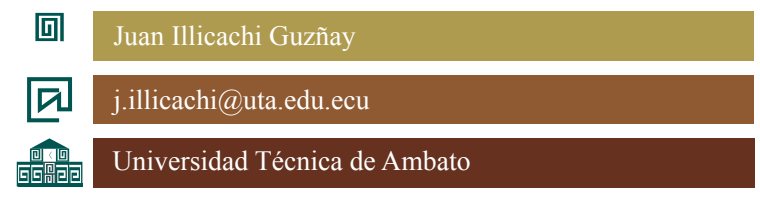

ORCID: https://orcid.org/0000-0001-6283-6290

\section{ABSTRACT}

The text analyzes (from the interviews, the specialized and secondary bibliographic review and other materials related to the subject) the transition of the Political Constitution of the Republic of Ecuador from 1998 to that of 2008. Making comparative reflections on the passage of a multicultural and multiethnic Constitution towards an intercultural and plurinational one. This passage implies the change of typology of Ecuadorian society, at least in theory, and how these categories and postulates constitute a toolbox for the insurgencies and dialogues of indigenous peoples and nationalities against the dominant structure. Next, it addresses the advances, limits and contradictions of constitutional practices and discourses. Both concepts: both interculturality and plurinationality seem to be underpinned as an ontological, epistemic and political counterhegemony. Epistemically, for example, they resurface or appear as local knowledge, although this knowledge becomes self-absorbed; that is to say, that they are only part of the subalternate population, beyond the incorporations that are made to them in the academies, but that is mostly marginal.

Keywords: Constitution, multicultural, multiethnic, plurinational, referendum.

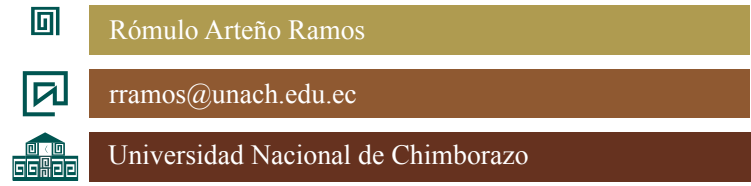

ORCID: https://orcid.org/0000-0001-9606-6546 


\section{KALLARI YUYAY}

Kay killkashka pankakunapika kay rimanakuykunmi awarishka: runakunapak makanakuy, mamakamachiypak ñankuna; shuk waranka, iskun patsak, iskun pusak watapi kilkachishka kamachiy; Ishkay waranka pusak watapi killkachishka kamachiy; kawsaypurapak mama llaktapi tikra yuyaykuna, shinallatak harkaykuanapash; shinallatak, tukuripiy churashka yuyaykuna.

Shuk waranka iskun patsak, iskun kanchis wata, aymuray killapimi - Ecuador mamallaktapika mama kamachiyta mushukyachinkapak hatun tantanakuyta wiñachinkapak ari mana nishpa tapuchirka, chaypika hatun charikkunata alikachik, shinallatak kullki sapa runakunami paykunapak nishkata rurarkakuna, manapash ñukanchik mañashkakunatami paykunapak allipaklla mama kamachiyta mushukyachishpa, manapash tukuykunata tapushpa ima shinami ishkay waranka pusak watapi mama kamachiyta allichishka shina allichirkakuna.

Shinallatakmi, shuk waranka iskunpatsak, iskun chusku wata, shuk punlla, kulla killapi Zapatista nishka awkakunaka, México, kiti San Cristóbal Chiapas llaktapi piñarishpa makanakuyta kallarichirka. Ishkantik hatun llaktakun, runakunapak hatun tantanakuykunamantami ñankunata pitishpa, shinallatak kiti llaktakunata muyuchishpa harkashpa, mana murukuta yaykuchishpa, sinchi wankurita rurashpa, llaktata pushakkuna paykunapak yuyashka, pacha mamapi tiyakkunata kichushpa llamkaytaka harkakkuna. Shinami, chay kimsa hatun tantanakuykunaka mama llaktakunawan chimparashpa yuyaykunata kushpa, shinchita makanakushpa kawsankapak wiñarinkuna.

Shinapash, shuk waranka iskun pastak, iskun pusak watapi killkashka hatun kamachiypika, kallari rikuchipika mushuk yuyaykunatami churarkakuna: kawsaypura, shinallatak tawka runakuna kawsayta rikuchiktami churarkakuna. Chay punchakunamantami kichwa runakuna, shinallatak yana runakunapash kamachyipika riksishka tukurka, shinallatak paykunapak allpakuna, kawsay, tantanakuy, ñawpa kawsay, ishkay shimipi rimashpa yachay, makanakuykunata runakunallatak allichinchunpash churarkakuna.

Shinami wakin makanakukkunaka ashallatapash kushiyarirkakuna, chay shuk waranka iskun pastak, iskun pusak watapi kallaripi churashka yuyakunamanta, shinapash, yachak runakunaka mana allikachinchu, ashtawanpash wakin uchilla kamaykunapika uchilla harkaykunata churashkamanta, kutin, Confederación de Nacionalidadades y Pueblos Indígenas del Ecuador (CONAE) tantanakuy, shinallatak Pachakutik tantanakushkakunaka chawpi chawpillapash kushiyarinkunami, ashtawanka ecuador mama kamachiypi runakunapak tawka kawsaykunata churashkamanta, kipamanka ñukanchik llaktapi tawka kawsak runakunaka ñami sumakta kawsashun yuyashpa. Chay tawka runakunapak aynikunata riksishpa hatun kamaypi killkashkakunaka kayshuk karu llaktakunapi killakashka shinami kan nishpa.

Pacha mama kasayta charikmi nishpa killkachishkaka maypipash mana rurashkata shuk mushuk yuyayta katishpa kullkita hapinatami rikuchin. Washaman tikrashpa rikukpika, ñukanchik pacha mamaka mana kunan shina kawsayukchu karka, ashtawanpash shuy hayllita shina runakunak ukupi kashkatami rikukkuna karka, kunanka Ecuador mamallaktapa kamachiypi pachamamaka kawsayta charinmi nikpika shuk yalli allí ruryami kan, maypipash mana shina yuyashkatami kunan punllakunaka churashkanchik.

Chay killkakpak yuyayta uchillayachishpa rikukpika, pachamamaka kawsayta charik, kawsaymantami rimarina, allí yachakkuna nishka shinaka, kawsayuk kashkamantami kaparikun, tawka watakunata colonialismo nishka llapishpa kawsashkamanta. Shinami pachamamaka Ecuador mamallaktapak kamachiypika runakunapak mañashkata paktachishka, shinapash kunan punllakunaka pacha mama kawsayta charinmi nikushpapash ecuador mama llaktata pushakka mana paktachikunchu, ashtawanpash ñami ñawpa yuyaypi shinallatak pacha mamataka kullkita hapikapaklla kashkata rikushpa allimantalla shuk hatun karu llakta rantikkunaman kukun.

Pacaha mama ishkay waranka pusak wata 
Ramachiypi aynikunaka punllantami pakishpa mana paktachin, (yana wirata karu llaktaman katushpa, yasunípi tiyak hillaykunata llushichishpa katushpa, shinallatak shuktanata rurashpami yallitak llakichiku); runakuna makanakuykunata harkashpa, kaparikkunata wichkachishpa, tantanakuykunata rurankapak mana ari nishpa, Pachamamata yanapakkunata chinkachishpa, mana allí kachishpa tukuykunata mana allí rimashpa, allí rikukpika, mana ima yuyaytapash kuypak, nimata mana alikachishpa, ima allitapash mana rurashpami llakichikun.

Rafael Correa, Ecuador Mama llaktata pushak kashpaka mana yankamanta, asirishpallachu rimarka, ashtawantakka runakunapak wawsayta, kawsaypurata, yuyaykunatami aharka, ñawpa salakuna runakunata llapishpa kawsakkuna shina. Correa Pushakka, yankata yachapashpami rimakkarka. Ñawpa salakunaka makashpa kamishpapash, kunan salalakunaka asishpa, kushiyashpa runakunata rimankuna, Correa shina. Correa nikkarka; pachamamapak wawallakuna nishpa, wawa runakuna, lluqui wawakuna nishpa, shinami kutin, mamam llaktapi kawsak hatun tantanakuyta pushakkunapash shukkunapash piñarishpa makanakuy kallarinkuna. Shinami, runakunataka, rakishpa, makanakuchishpa, wakllichishpa runakunapak tantanakuykunataka llakiman aparka.

Chashna makanakushpa kallarishpaka mana ima allíkunawan rinkunachu, ashtawanpash chay mama kamachiypi killkashka kawsaypura, tawkakuna shuk shinalla kawsayta shinchiyachinapak rantika makanakuytami kallarichin, chasnalla katishpaka ima alita mana rurakushkatami rikuchin, kutinllatak ñawpa kamachiypi rurashka shinallatak nimatapash mana mushukyachishpa apakushkatami rikuchin.

Shuk waranka iskun patsak, iskun pusak watapi mama kamachiypi churashka tawkakawsay, achka kawsay, shinallatak ishkay waranka pusaku watapi mama kamachiypi churashka shukshinalla kawsaypura, shinallatak kawsaypura yuyakunata churashkaka yalli mana kallpak shina runakunapak llaki kawsaytami harkashka. Shinallatak shuy yuyaypika kutin allí yuyarishpa, allí rikushpa runakunapak kawsayta wiñchinatami rikuchin, shinallatak, yachay ukumanta rikukpika runakunapak sumak kawsaymi rikurin, tantanakushka runakunapak aynikunamanta, kawsaypuramanta paykunapaklla allí kashkata rikuchikmi kan, shinapash mayhanpash chay yuyayta charishpaka chakrurik shinami tantalla llamkankuna.

Shuk allí yuyachiyka kawsaypurata rikukkunata wiñachina mutsuriymi, hatun yachana wasikunapi katiy rikuytami wiñachina, ima shina chay kawsaypuramanta, shinallatak shukshinalla kawsaypura hawa llamkaykunata kamayta rurachunkuna, chay llamkaykunataka mamallaktapak kullkiwanmi yanapana kan, allí imatapash rurarichun nishpaka.

\section{YACHAYÑANA}

Kay killkayta rurankapakka tawka yachayñanakunami yanaparka: Kamachikunamanta yachak warmikunata, karikunatami tawka tapuykunata rurarkanchik. Ashka kutichishkakunami kay killkashka wachukunapika tiyakunkuna; kay killkashkata imashinami ñawirishpa rikunkakuna.

Shinallatak, kamukkuna, shuktak killkashka pankakunata ñawirishpami kay llankashka pankakunaka rurarirka. Yuyaykunapurata chimpapurachishpa, rimarishkakunata wankurichishpa, piñanakuchishpa, awachishpami, kay killkayka rurarishka.

Maypika, kay killkaypika chaymi waklli wakllilla, alli rikushkakunatami kay ki1lkaypika yuyaykuna awarishka.

Ashtawantakka, kay kinmsa yachayñanami tinkirishpa killkaytaka yanapashka. Yuyaykuna, chimpapuranakuypika killkakpak yuyaypashmi rikurin.

Kay killkashka yuyaykunaka imapakla allikashkatami willan, shinallatak imalla illashkatami rikuchin. Pillatak, mama kamachikwanka alli kawsayta charina kashkatami riluchin. Kay tapurikunapimi shuktak tapuykunata rurarkanchik. Ima kamachikkunallatak runakunapakka allli, allitak kawsayta kushanin; Mamakamachikka tukuy runakunatachu ukllay, kuyanpash; Sumak kawsa- 
ypi purinkapakka pillatik llankanakanchik; kamachikkunapi nishkashinachu kawsakunchik, mana kashpaka, ruraywan, yuyaywan rakirishkachu kan. Ashtawantakka kay tapuykunami purirka mana runakunata tapunkapaklla, shinapash, kamukkunapipash ñawirishpa tapurinkapak.

Shinapash, kay, yachayñanata purichinaka sinchimi kan. Ñawpa pachakunapika, yurak runakunaka, nishunpash, shuktak llaktamanta shamuk runakunaka, ashtawantakaka, Abya-Yala, llaktapi kawsakkunataka mana yachankichikchu, ñukanchikmi yachanchik nikmi karkakuna. Mashna yachaskapash mana usharirkachu, yuyaypash chinkarirkami, kawsakunapash wañurkami, runakunata, pushakkunatapash wañuchirkakunami. Nawpa taripak, llakichik, yurak amawtakunaka kapakmanmi katurirkakuna.

Chashna taripanaka, kay pachakunapika, sinchitami rimashka kashka: maypika paykuna puralatak, maypika wakchakunamanta. Kunan pachapika, tapukunapish yachanmi, tapushka runakunapash yachankunami, tukuykunamu yachankuna. Maymanta shamuk taripakkunapash shukta yuyayta, shuktak kawsayta, shuktak yachayta, shuktak runakunata paypak kawsayta shinllatakmi kuyana kan. Mana, taripakpak kawsay shinachu kan nishpaka, shuktak kawsaykunata llapinallachu kan.

Taripayta rurak runakunaka, mana, paykunapak kamukkunata killkashpallachu sakirina kan, ashtawankari, maypi tapurishkapak kawsayta allichishpa ñawpakman apanami kan. Taripakkuna alli kawstami mashkana kan. Yachakkunapak, killkakkunapak karipak, warmipak, yuyakkunapak, wawakunapak kawsaykunapimi wankurina kan.

Kay killkashka pankakunaka mana runakunapak kishpirinatachu karan, shinapash ashaku yuyayakunawanmi yanaparin. Shinapash, ñawpa yachayñanataka shakishpami, shuktak yachayñanata ushashkanchik. Chay yachayñanaka chinpapura yachaymi kan, wankurinakuhspa killkaymi kan. Yachayñana minka nishpa tukuchishun. Kay Yachayñanamanataka shuktak killkakunapi ashtawan yuyarushpaka allichari kanman.

\section{RIKUSHKA NAKUY \\ CHIMPAPURA-}

1. RUNAKUNAPAK MAKANAKUY, MAMAKAMACHIYPAK ÑANKUNA

Ecuador ama llaktapi kawsak runaka mana hawallachu kawsaypak mutsuykunatka charishkakuna, ashpawampash sinchita makanakushpa, hatun tantanakuykunawan wankurinakushpami mañaykunataka charikkuna kashka, kunan punllakunakamami Abya yala tantanakuykunawan minkarishpa makanakunkuna. Shina: Abya yala hatun llaktapika, ishkay makanakuykunami tiyashka rikurin. Ecuador Mamallaktapi shuk waranka iskun ptsak, iskun chunka watapi shuk hatun runakuna hatari makanakuy tiyarka, kutin, chay watakunallatakmi Bolivia llaktapipash runakunaka tukuy ñankunata harkashpa llaktata pushakkunawan makanakuy tiyarka. Chay ishkay ruraykunaka makanakunkapak mushuk tantanakuykunata wiñachishpa wankurishpa llaktata wichiman pushana kashkatami rikuchin "chay hatun ruraykunataka kunan kawsaypak yachanami tukun" (García 2003:195).

Shinallatakmi, shuk waranka iskunpatsak, iskun chusku wata, shuk punlla, kulla killapi Zapatista nishka awkakunaka, México kiti San Cristóbal Chiapas llaktapi piñarishpa makanakuyta kallarichirka (Wallerstein 2008). Ishkantik hatun llaktakuna (Bolivia shinallatak Ecuador), runakunapak hatun tantanakuykunamantami ñankunata pitishpa, shinallatak kiti llaktakunata muyuchishpa harkashpa, mana murukuta yaykuchishpa, sinchi wankurita rurashpa, llaktata pushakkuna paykunapak yuyashka, pacha mamapi tiyakkunata kichushpa llamkaytaka harkakkuna. (Gutierrez 2009: 95). Shinami, chay kimsa hatun tantanakuykunaka mama llaktakunawan chimparashpa yuyaykunata kushpa, shinchita makanakushpa kawsankapak wiñarinkuna.

Wakinpika, runakunapak tantanakuy mañashkakuna, makanakuykunaka mana Mamallaktapak ruraykunata wakllichishpa panta mañaykunachu kan, ima shinami Teresa Sie- 
rra chay guerrero llaktapi chapak hawamanta rikushka shinaka:

Shuk waranka, iskun patsak, iskun chunka, pichka watamantami, uchillallaktapi tantanakushpami yuyarikkuna, wañuchina illapa hillaykunata chay Costa Chica, Guerrero chusku chunka, pusak Awkakunata pushakkuna ukupi rikuchishpa killkachirkakuna. Shinami chay llaktapi chapakkunaka mana mamallaktapak tikraman ruraykunata rurana kashkata rikuchin, ashtawanpash shuway llakikunata wankurishpa allichinkapakmi kan, mana mamallakta allichi tukushkamanta. (Sierra 2009:14)

Ecuador mamallaktapi wiñachishka runakunapak hatun tantanakuypash mana tikraman llaktata llakichinkapakchu kan. Tukuy ruraykuna, shinallatak mañaykunapash kamaykunata paktachishpami kawsankuna. Wakinpika, shina paktachikunaka runakunata muspachinkapakmi kan, ama piñarishpa mañaykunata rurachun, shinami makanakuk runakunata mana makanakuk runakunata rurankuna (Poole 2008). Mamallaktamanta chashna muspachisha nikpipash, runakunapak hatun tantanakuykunaka uramanta hanakmanta makunakushpami paktachiykunta shinallatak mañaykunatapash hapishkakuna, shina: mushuk kamachiyta rurachirka.

\section{SHUK WARANKA, ISKUN PATSAK, ISKUN PUSAK WATAPI KILKACHI- SHKA KAMACHIY}

Shuk waranka, iskun patsak, iskun kanchis wata, ishkay chunka pichka punlla, aymuray killapimi Ecuador mamallaktapika hatun tapuy tiyarka, tukuy kiti llaktakumanta tantanakushpa hatun kamachiyta kutin allichishpa killakankapak. Shinami, chay shuy waranka iskun patsak, iskun kanchis wata, ishkay chunka punlla, kayak killapi, llaktapi kawsakkuna ari nishkawan kamayta killka kallarirkakuna, chay llankaytaka ñawpa pushak Oswaldo Hurtado aparka, kipamanka Luis Mejía Montesdeoca mishumi chay tantanakuytaka pusharka.

Kaytami yuyarina kanchik, llakpi tukuy kawsakkunaka tantanakushpa kamayta alli- chinmi ari nirka, shinapash, achkata charik tantanakushka millay mishukuna tantanakushpaka paykunata nishkatami rurarkakuna, ashtawanpash mana ari nishkakunakamami tukuyta kamachiytaka mushukyachirkakuna. Shinami, chay kipaka shuk waranka iskun pastak, iskun pusak wata, pichka punlla inti killapi, Riobamba llaktapi mana ni pitapash tapushpalla hatun mushukyachishka kamachiytaka karwa killapi, chay watallatak ña ari nishpa churarkakuna.

Shinapash, shuk waranka iskun pastak, iskun pusak watapi killkashka hatun kamachiypika, kallari rikuchipika mushuk yuyaykunatami churarkakuna: kawsaypura, shinallatak tawka runakuna kawsayta rikuchiktami churarkakuna. Chay punllakunamantami kichwa runakuna, shinallatak yana runakunapash kamachyipika riksishka tukurka, shinallatak paykunapak allpakuna, kawsay, tantanakuy, ñawpa kawsay, ishkay shimipi rimashpa yachay, makanakuykunata runakunallatak allichinchunpash churarkakuna.

Shinami wakin makanakukkunaka ashallatapash kushiyarirkakuna, chay shuk waranka iskun pastak, iskun pusak watapi puntapi churashka yuyakunamanta, shinapash Agustín Grijalva yachakka (2008) mana allikachinchu, ashtawanpash wakin uchilla kamaykunapika uchilla harkaykunata churashkamanta, kutin, CONAIE tantanakuy, shinallatak Pachakutik tantanakushkakunaka chawpi chawpillapash kushiyarinkunami, ashtawanka ecuador mama kamachiypi runakunapak tawka kawsaykunata churashkamanta, kipamanka ñukanchik llaktapi tawka kawsak runakunaka ñami sumakta kawsashun yuyashpa. Chay tawka runakunapak aynikunata riksishpa hatun kamaypi killkashkakunaka kayshuk karu llaktakunapi killakashka shinami kan nishpa: Colombia 1991, Perú 1993, Bolivia 1994, Venezuela 1999 (Becker 2015).

Ecuador mama llaktapi tiyak hatun runakunapak tantanakuykunaka, chay shuk waranka iskun pastak, iskun pusak watapi killkashka kamachiyta pushakkuna mana pakchikta rikushpaka, kutinmi shinchita mañaykunata rurashpa makanakuykunawan kallarirkakuna. Shuk waranka iskun pastak, iskun pusak 
watapi killkashka kamachiyka achka allí yuyaykunatami charin, rimaykunata, aynikuna, hanpirinamanta, yachaymanta, ashtawanpash ishkay waranka pusak watapi killkachishka kamachiyka ñawpa kamachiypi churashka yuyaykunata sinchiyachishpami killkachin.

\section{ISHKAY WARANKA PUSAK WATA- PI KILLKACHISHKA KAMACHIY}

Rafael Correa Delgado, allí yachak,llaktata pushankapak mushuk yuyayta charik, allí yachachaik, amawta, kullki kamayuk, Alfredo Palacio pushakuy watakunapimi riksishka tuparka, ishkay waranka sukta watapimi Ecuador mamallaktapak pushak tukurka. Pichka chunka, sukta chunka kanchis aspiwanmi $(56,67 \%)$, Ishkay chunka punlla, sasi killapimi chay achka kullkisapa Alvaro Noboa ishkay kutin mishanakuypi misharka, chaypak washaka ishkay waranka kanchis wata, chunka pichka kulla killapimi ña llaktata pushankapak kallarirka.

Tukuymanta yalli paypak yuyay churaskaka mamallaktapak kamachiyta mushukyachichinkapak hatun tantanakuyta wiñachinatami rurasha nishpa yaykurka, shinallatak runakunata hatun tantankuy pushakkunawan, kayshuk tantanakuyta pushkunawanpash, llukimanta yuyayta charik tantanakuynata tantachishpa pushanatami kallarirka. (Partido Socialista, Pachakutik, Movimiento Po- pular Democrático, Izquierda Democrática, Partido Roldosista Ecuatoriano).

Shinami, Rafael Correa Ecuador Mamallaktata pushak yaykushpaka, ishkay chunka pichka punlla, kulla killa, ishkay patsak kanchis watapi hatun tapuyta rurankapak kayachirka. Kimsa killa washa, pay pushankapak yaykushka kashpami kayachirka ari mana ninchunkuna, shinaka, chunka pichka ayriwa killa, ishkay patsak kanchis watapi ña kayachikpika ecuador mamallaktapi tukuy kawsakkunaka pusak chunka yupaykunawan (80\%) ari, mushuk kamachiyta killkachun shuk tananakuyta wiñachichun nishpa mishachirka (Becquer 2015). Ña, kimsa chunka punlla, kuski killa, ishkay waranka kanchis watapi kayachikpika patsak kimsa chunkamantaka, pusak runakuna paypak wiñachishka llaktata pushaypak ukumanta muisharka, chasnami chunka shuk marka llaktakunamanta misharkakuna.

Kutin, shamuk wata, ishkay chunka pusak punlla, sasi killa, ishkay patsak wata kayachipika sukta chunka kimsa, iskun chunka kimsa aspikunawanmi ari nirkakuna, kutin, ishkay chunka pusak, chunkakunallami mana nirkakuna, mana allí aspishkakunaka kanchis, ishkay chunka kimsakunatami aspirkakuna, Guayaquil llaktallapimi mana nishpaka misharka, (chusku chunka, iskun kanchis yapaykunawan mana nirka, shinallatak, chusku chunka, sukta pusaku yupaykunaka ari nirka).

\section{Killkak rurashka shuyu}

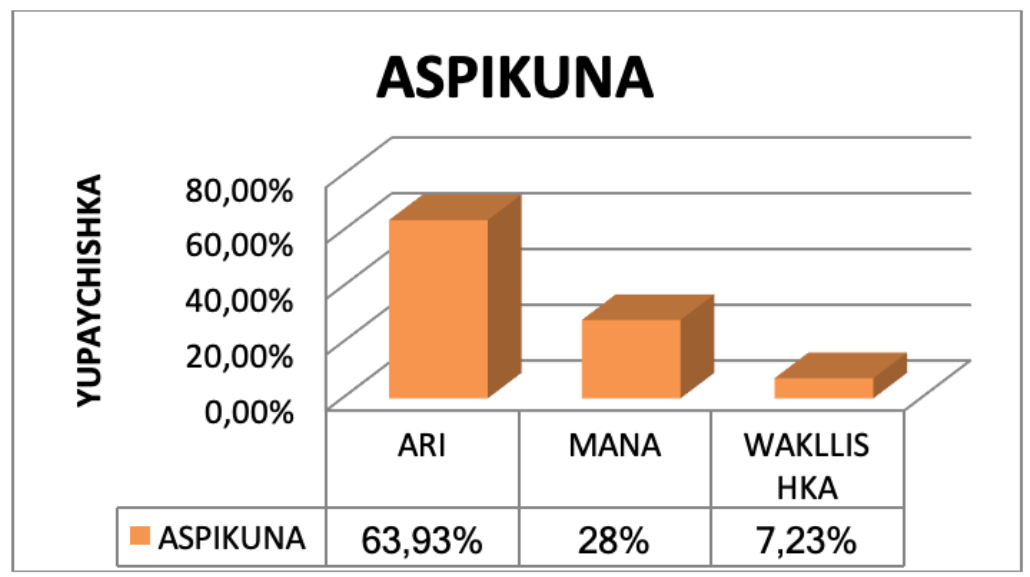

Shuyu 1: Shuyupi akllashka yupaykuna 
Kay suyushkakunapika (1 y 2 ) imashina Mama Llaktapi pushakkunata akllashkakunatami rikuchin: arinishkakuna (63, 93\%), mananishkakuna (28\%), sinallatak wakllishkakunatapash (7,23\%). Nukanchik yuyaypika, kay yupaykunaka mamallaktapak akllaykunapak tandanakuyka allí kawsaytami rikuchin. Astawantakka, kay 63,93\% arinishka yupaykunami Ecuador, Mama Llaktapi allí tantanakushkata rikuchin. Mama Llaktata pushakpahs sinchi sinchitami shayarishpa yuyarishpapash pushanka. Shinaka, karipak, warmipak, wawakunapak, yuyakkunapakmi allí kawasaykunakanka.

Chay hatun kayachiypi mishayka, runakunapak hatun tantanakuy yanapashpa llankashkamantami misharka. mana Correa pushakllachu mishashka, runakunapak tantanakuy wankurishkakunami mishan nishpami Ana María Anaguarqui Chimborazo markamanta runakunapak tantanakuy pushkka nirka. Ima shinami Venezuela llaktapi waranka iskun patsak, iskun chunka iskun watapi killkashka kamachiy shina, ñukanchik Ecuador mamallakta kamachiyka tukuytami ukumanta mushuk yuyaykunta churarkakuna, chasnami mushuk pushakkunatapash akllana tukurka, kiti llaktamanta kallarishpa, kamaykunata rikukunata, shinallatak mamallaktata pushakkama, (Becker 2015). Achka kawysakkunamanta shinallatak tawka runakunamanta, shuk kawsaypura, shinallatak tawkakuna shukshinalla kawsayta charishkata rikuchik yuyayta churaskami ashtawanka sumak allí karka.

Shuk niki.- Ecuador Mamallakta mamakamachiy hayñi, kishpiriyshka runakunapak, llakta-ushay, hawaman awkiyak, kikinlla, tantachik kawsaypura kawsay, tawka kawsayyuk, mana iñipash kikinyashka Mamallaktami kan, Kay hatun llaktaka Mamalakta shinami tantarin, shinapash markakunapi. llaktakunapi, karumanta kamachishpami pushan. Mamallaktaka awkikayka llaktapimi minkarin, kamachikka paykunapak munaypimi sapiyarin, runakunapaktantanakuy ushaywan paktachin, paykunapak yachaywan chaypi kashpa
Mamakachiypi ima shina ikta hawaman churashpa pushan. Mamallaktapak allpa llaktapi tiyakkuna mana kutin wiñarik kakta, mamallaktapak charishkata, mana shitanalla, mana pipash yaykunalla, mana katunalla, wiñaypak charinallami kan. (Becker 2015: 120).

Kay yuyayta churashkaka, Ecuador mamallakatapi tawka kawsaypura, chunka kimsa llakta kawsay, shinallatak yalli pachaka mishukunawan tantalla kawsayta rikushkatami umanachin. (Trujillo 2012; Becker 2015) shinallatak kimsa yuyaykunatami rikuchin

(Shuk) Ecuador mamallaktapika tawka tantanakushka runakuna kawsashkata, ashtawanpash mana shuklla tantarishka runakunallachu kawsan nishpa riksirishkatami rikuchin. (ishkay) Shuy sumak yuyaypika, tukuykuna sumakta kawshuk chayshuk wankurishpa mana makanakushpalla shinallatak mana kayshukka hatun tukushpalla makipurashpa kawsachun yuyaytami rikuchin. (kimsa) Ña kipawanka, shuk tantanakushka runakulla, kayshukkunata mana alikhishpa kawsanata kunkarichun nishpa hatun yuyaytami rikuchin (Ávila 2012:283).

Shinamari, kawsaypura rikuchiyka tukuy kawsaypi mamallaktapak kamachiy mutsurishka kana kashkata rikuchin, kay yuyakunaka allí kawsaypi, mama llaktapak kallaripi, shinallatak tukuy mama kamachiypimi rikurin (Ávila 2012). Katherine Walsh rikuypika (2009-2012) chay sumak yuyaykunaka, ñawpa runakunapak kawsaymanta hapishkakunami kan, paykuanaka mamallaktamanta chikanchishka, waklichishkami kawsakkuna karka.

Kutin, paykunapak sumak yuyaykunata mama kamchiypi churashpa killkashkaka aschka allimi kan, chaymantami llaktata pushakkunaka paykunataka sinchiyachishpa sumakta tantalla pushana (Grijalva 2008). Llaktata pushak Rafael Correa, mana tantalla tawka runakuawan ecudor mamallaktata pushashkamantami runakunaka piñarishpa chinkanyarishpa anchurkakuna. Ecuador 
mamakamachiypika shuk sumak rikuytami churarkakuna pachamamapak aynitami killkachirka, kaykamaka pachamaka shuy haylli shinallami rikushka karka, kunanmanta ñawpakmanka pachamamaka kawsayta charikta rikushkami kan (Acosta 2014:323).

Pacha mama kasayta charikmi nishpa killkachishkaka maypipash mana rurashkata shuk mushuk yuyayta katishpa kullkita hapinatami rikuchin. Washaman tikrashpa rikukpika, ñukanchik pacha mamaka mana kunan shina kawsayukchu karka, ashtawanpash shuy hayllita shina runakunak ukupi kashkatami rikukkuna karka, (Cartesinopak yuyay), kunanka ecuador mamallaktapa kamachiypi pachamamaka kawsayta charinmi nikpika shuk yalli allí ruryami kan, maypipash mana shina yuyashkatami kunan punllakunaka churashkanchik (Walsh 2012).

Chay killkakpak yuyayta uchillayachishpa rikukpika, pachamamaka kawsayta charik, kawsaymanta rimarina, Hinkelammert nishka shinaka, kawsayuk kashkamantami kaparikun, tawka watakunata colonialismo nishka llapishpa kawsashkamanta. Shinami pachamamaka ecuador mamallaktapak kamachiypika runakunapak mañashkata paktachishka, shinapash kunan punllakunaka pacha mama kawsayta charinmi nikushpapash ecuador mama llaktata pushakka mana paktachikunchu, ashtawanpash ñami ñawpa yuyaypi shinallatak pacha mamataka kullkita hapikapaklla kashkata rikushpa allimantalla shuk hatun karu llakta rantikkunaman kukun.

Pacaha mama ishkay waranka pusak wata kamachiypi aynikunaka punllantami pakishpa mana paktachin, (yana wirata karu llaktaman katushpa, yasunípi tiyak hillaykunata llushichishpa katushpa, shinallatak shuktanata rurashpami yallitak llakichiku); runakuna makanakuykunata harkashpa, kaparikkunata wichkachishpa, tantanakuykunata rurankapak mana ari nishpa, Pachamamata yanapakkunata chinkachishpa, mana allí kachishpa tukuykunata mana allí rimashpa, (Sierra 2014:36), allí rikukpika, mana ima yuyaytapash kuypak, nimata mana alikachishpa, ima allitapash mana rurashpami llakichikun (Ospina 2009).
Shinallatak, ñukanchik, Mamallaktata pushakkunaka; pi kashpapash, maykan kashpapash, wiñay kawsaypimi runakunataka llakichishpa, wañuchishpa, shamushkakuna. Shina, runakunapak kawsay kakpipash, manatak llakillachu kawsashkakuna, imalapash, imashinapsh pukllaywan, ushaywan, munaywan, yachaywanmi ñawpakman katikmushkakuna.

Chasna makanakushpa kallarishpaka mana ima allíkunawan rinkunachu, ashtawanpash chay mama kamachiypi killkashka kawsaypura, tawkakuna shuk shinalla kawsayta shinchiyachinapak rantika makanakuytami kallarichin, chasnalla katishpaka ima alita mana rurakushkatami rikuchin, kutinllatak ñawpa kamachiypi rurashka shinallatak nimatapash mana mushukyachishpa apakushkatami rikuchin.

\section{KAWSAYPURAPAK MAMA LLAK- TAPI TIKRA YUYAKUNA, SHINALLA- TAK HARKAYKUANAPASH}

Kaysaypurata shinallatak shukshinalla tawka kawsaykunata shuk mamallaktapi churashkaka shuk "hayllikuna shinami kan" (Foucault 2010), shuktakkunapak kawsaykunata riksinkapak, kipanchishpa pakashka kakpi rikurinapak, shinallatak pakalla kawsakushta rikuchinkapak. Kawsaypurayuk mamallaktapika wakchalla llakilla kawsanaka mana pakashka kanachu (Ávila 2012). Shuktakkunapak kawsaykunata riksinaka, sumakta yachana kikin yachaskata tikra kunkarishpa yachaypakmi kan, chay hatun tantanakushka runakuna, kayshuk uchilla tantanakushka kawsaykunata yachanaka achka mutsurishkami, tantalla llamkana, ñukanchik kiwakunallawantak hampirinamanta, warmikuta alikachishpa kawsaymanta yachanakuna, kutin, kawshuk hatun llaktakunapak kawsaykunaka achka yankamanta wañuykuna, shinallatak shuk hampikunawan hampirishpa kawsaytami apamushka (Ávila 2012). Shina yachaykunataka, kashnami shutichinchik kawsaypura yachay. Shimami kay mutsurinamanta rikukpika yachay pampakunapi, shinallatak yachana ukukunapipash kutin allí yachachina kanchik, kawsaypura shukshinalla tukushpa yachaytami ruray tukunchik (Walsh 2013), chasshna millay yuyay ñukanchik ukupi ti- 
yashpa hatun tukuna yuyayta, shinallatak pitapash mana alikachishpa kawsana yuyayta kukpi kunkarinkapak (Foucault 2014:16).

Shinami, kay mamallaktapi kawsaypurata sinchiyachinaka mana shuk yanka rimaylla kay. Paktaykunaka mana kamaykunapa killkashkallachu kana kan, yachay wasipi wawakunata yachachinkapaklla, hatun pushayta wiñachishpalla, karu rikuypi rikurinkapakllachu kana kan. Ashtawanpash mamallakatapi kawsaypuramanta rimayka hatun paktaykunapakmi, tukuy runakuna wankurinakushpa sumakta kawsankapakmi kan (Ávila 2012:378).

Hatun yuyarina, tapuy punchakunapimi kawsanchik, ashalla waklishka yuyaykunata kushpami kawsanchik (De Sousa Santos 2010) mana killkana pataku washamanta sumakta rimashpalla makipi waktayta chaskinkapakllachu kan, "imallatapash ashtawanmi rurana kanchik" (R. M. Illicachi, comunicación personal, 18 de enero de 2019).

Kutinllatakmi yuyachini, kawsaypuramanta llankankapakka yachay ukumantami kallarin, yachana wasi ukumantami wiñachinaImatatak yachana wasika wiñachin- Yachana wasika kawsaypura wankurishpa kawsayta, mana kashpaka sapalla kawsanatami yachachi tukun. Yachana wasi ukupimi rikchachi, mana kashpaka kawsaypuramanta, shukshinaklla kawsayta chikakachichun yachachin, chaymantami tawka llamkaykunata rurana kanchik, shina: yuyaykunata chimpapurashpa tantanakuy, killkanata killkakatishpa, yachana kamukunata killkashpa, sumak kawsaymanta, tantalla kawsaymanta shukkunamantapash. Kawsaypuramanta, shukshinalla kawsaymanta, twka tanatanakuy kawsaymanta, shukshina kawsaymanta rikunkapak killkakunaka achkakunami tiyan, shinapash imashina yachay ukunamanta llamkanaka mana achkachu rikurin (Martínez 2007).

Kawsaypuramanta, shukshinalla kawsaypuramanta, sumak kawsaymanta hatun yachana wasikunapi, uchilla yachana wasikunapi yachachiyka mana kichwa runakunapakllachu kana kan, ashtawanpash tukuykunapakmi minkashka kan. Mamallaktapi kawsaypura, shukshinalla kawsaypurata sinchiyachinkapakka tukuylla yachanana wasikunapi, shinallatak tukuy yachaykunapimi yachachina kan, "yachankapakka ñawpaman yachaskata kunkarishpami yachana" (Walsh 2013), "chimpapurashpa wankurishpa rurayta katinami allí kanman yuyanimi" (Foucault 2014:14), mana kawsaypura ima kashkata yachankapaklla, ashtawanpash kawsaypak ima allí pushaypak, mana makanashpa kawsaypak, mana alikachishpa kawsayta sakishpa sumakta shuk shinalla tawkakuna kashpapash kawsankapak.

Karu llaktapi killkachishka kamchiykunapash llamkaymanta pushak 169, muyuntik llaktapak killkashka UNESCO nishka shinaka, sinchitami llankana kan, ecuador mamallaktapika manatak ayllu ukupi yachay, llaktakunapi, apunchikta yachana wasi ukukunamanta chikanchishpa llankanachu, ashtawanpash anta uyarianta, yachana wasikunamanta tukuy manyakunamantami kawsaypurata, shukshinalla kawsaypuratka yachachina kanchik mana shina llankakpika sumak rimashka, allí kamachiyta charishkata rikuchikllami sakirinka.

Kawsaypura yachayta mana hawamanta churashkalla kachunka, ayllumanta wiñachishkami kana kan, paykunami ñawpa llaki kawsaytaka yachankuna, paykunami rimanakushpa kawsanataka mañankuna, shinami kikinyarishpa shukshina yuyashpapash kawsanata charinkuna (Ávila 2012:304), runakunapak hatun tantanakuy, yana runakunapak tantanakuy, shinallatak llukichishka yuyayta pushakkuna sinchita kaparishpa mushuk kawsayta wiñachinkuna, paykunaka mana pushak tukunkallapak, mañaykunata makanakuyta pushankapakllachu llankankuna, ashtawanpash kunan pachakunaka runakunapak tantanakuykunaka sumak kawsaypak allí yachaykunata yuyarinakushpa churankapak, ima shina kawsaypurata yachay ukukunamanta churankapakmi kankuna (Escobar 2001), shinami, kichwa runakuna, yana runakuna, mishukunapash wankurinakushpa shukshinalla tawka yuyakunawan llaktata sinchiyachiyachinkakuna.

Kay yuyaypimi awarin, Norma Bayas rimarishka: "Nukanchik kamachikunaka, tukuykunatami kuyaya kan, pita mana llakichinachun kan. Kunan pachaka, ñawi allí kawsana kanchik, chimpapura rikunakushpa, makita kunakushpa, shunkuwantak kuyanakushpa. Mana shunkumanta kuyanakukpika ima allí kamachikkuna tiyakpipash mana, imapak mana ushunkachu" (N. Bayas, comunicación personal, 15 de marzo de 2019). Chashna kakpika, nipakmi kanchik, kamachikkunaka allimi tiyashka; shinaka, kamachikukunata purichikkunami waklli, waklli kashkakuna. 


\section{TUKURIPIY CHURASHKA YUYAYKUNA}

Shuk waranka iskun patsak, iskun kanchis wata, aymuray killapimi - Ecuador mamallaktapika mama kamachiyta mushukyachinkapak hatun tantanakuyta winachinkapak ari mana nishpa tapuchirka, chaypika hatun charikkunata alikachik, shinallatak kullki sapa runakunami paykunapak nishkata rurarkakuna, manapash ñukanchik mañashkakunatami paykunapak allipaklla mama kamachiyta mushukyachishpa, manapash tukuykunata tapushpa ima shinami ishkay waranka pusak watapi mama kamachiyta allichishka shina allichirkakuna.

Shuk waranka iskun patsak, iskun pusak watapi mama kamachiypi churashka tawkakawsay, achka kawsay, shinallatak ishkay waranka pusaku watapi mama kamachiypi churashka shukshinalla kawsaypura, shinallatak kawsaypura yuyakunata churashkaka yalli mana kallpak shina runakunapak llaki kawsaytami harkashka. Shinallatak shuy yuyaypika kutin allí yuyarishpa, allí rikushpa runakunapak kawsayta wiñchinatami rikuchin, shinallatak, yachay ukumanta rikukpika runakunapak sumak kawsaymi rikurin, tantanakushka runakunapak aynikunamanta, kawsaypuramanta paykunapaklla allí kashkata rikuchikmi kan, shinapash mayhanpash chay yuyayta charishpaka chakrurik shinami tantalla llamkankuna.

Shuk allí yuyachiyka kawsaypurata rikukkunata wiñachina mutsuriymi, hatun yachana wasikunapi katiy rikuytami wiñachina, ima shina chay kawsaypuramanta, shinallatak shukshinalla kawsaypura hawa llamkaykunata kamayta rurachunkuna, chay llamkaykunataka mamallaktapak kullkiwanmi yanapana kan, allí imatapash rurarichun nishpaka (García 2007:85).

Ecuador mamallaktapak ishkay waranka, pusak patask watapi killkachishka kamachiypi kawsaypura yuyay ñan shina kakpika, tukuy yachaykunapimi churana kan, uchilla shinallatak hatun yachaykunakama, tukuy markakunapi, shinami kawsaypura llamkayka mana shuk pachalla, wakin yuyarishka pachalla yachachinka, mana runakunapakilapash kanka, ashtawanpash tukuykunapakmi kanka.

Ecuador mama llaktaka, tukuy kamachiykunapi killkashkanataka shina, kawsaypura, shukshinalla kawsaypura, sumak kawsay, piñanakuy allichiykunataka paktachinatakmi kan, runakunapak hatun tantanakuykunawan wankurishpa ima shina kawsaypura yuyayta paktachinakunatami rurana kan.

\section{KIMIRISHKA KAMUKUNA}

Acosta, A. (2104). El Buen Vivir como alternativa al desarrollo: reflexiones desde la periferia de la periferia. En José Luis Coraggio y Jean Louis Laville (eds.) Reinventar la izquierda en el siglo XXI (p.p. 317-320). Quito: Instituto de Altos Estudios Nacionales.

Ávila, R. (2012). ¿Debemos aprender el derecho penal estatal de la justicia indígena? En Boaventura de Sousa Santos y Agustín Grijalva (eds.). Justicia indígena, plurinacionalidad $e$ interculturalidad en Ecuador (pp. 279-304). Quito: Abya-Yala.

Becker, M. (2015). ¡Pachakutik! Movimientos indigenas, proyectos y politicos y disputas electorales en el Ecuador. Quito: Abya-Yala.

De Sousa Santos, B. (2010). Descolonizar el saber, reinventar el poder. Montevideo: Gráfica Don Bosco.

Escobar, A. (2001). Introducción: lo cultural y lo político en los movimientos sociales latinoamericanos. Arturo Escobar y Sonia Alvares (eds.). Politica cultural, cultura politica. Una mirada sobre los movimientos sociales latinoamericanos (pp. 15-50). Bogotá: Instituto Colombiano de Antropología.

Foucault, M. (2014). Las redes del poder. Buenos Aires: Prometeo Libros.

García, F. (2003). Política, Estado y diversidad cultural: A propósito del movimiento indígena ecuatoriano. En Víctor Breton (ed.). Estado, etnicidad y movimientos sociales en América Latina (pp. 193-204). Barcelona: Icaria Editorial.

García. F. (2007). Los pueblos indígenas del Ecuador: derecho y bienestar. Informe alternativo sobre el cumplimiento del Convenio 169 de la OIT. Quito: Flacso-Ecuador.

Grijalva, A. (2008). El Estado Plurinacional e Intercultural en la Constitución ecuatoriana. Ecuador Debate, 75, 49-62.

Gutierrez, R. (2009). Los ritmos de Pachakutik. Levantamiento y movilización en Bolivia (20002005). México: Bajo Tierra.

Martínez, A. (2007). Derechos de los pueblos indígenas del Ecuador. Quito: Abya-Yala.

Sierra, T. (2009). Entre la legitimidad y la ilegalidad: las apuestas de la policía comunitaria. Multiculturalización del estado, derechos indígenas y la globalización en América latina. Congreso 53 de Americanistas llevado a cabo en la ciudad de México. 
Sierra, N. (2014). Las falsas promesas de la Revolución Ciudadana: Excluyendo el contenido comunista de la revolución socialista. El correísmo al desnudo. Segunda edición (pp. 134144).Quito: Montecristi Vive.

Trujillo, J. (2012). Plurinacionalidad y Constitución. En Boaventura de Sousa Santos (ed.) Justicia indígena, plurinacionalidad e interculturalidad en Ecuador (pp. 305-316). Quito: Abya-Yala.

Ospina, P. (2009). Movimientos sociales y participación política. En Ángel Montes (ed.). Ecuador contemporáneo. Análisis y alternativas actuales (pp. 199-222). Murcia: Universidad de Murcia.

Poole, D. (2008). El estado y sus márgenes. Etnografías comparadas. Cuadernos de Antropología Social, 27, 19-52.

Walsh, K. (2013). Pedagogías decoloniales. Prácticas insurgentes de resistir, (re) existir y (re) vivir. Tomo I (editora). Quito: Abya-Yala.

Walsh, K. (2012). Interculturalidad crítica (de) colonialidad. Serie pensamiento decolonial. Quito: Abaya-Yala.

Wallerstein, I. (2008). ¿Qué es lo que los Zapatistas han logrado? Contrahistorias. La otra mirada de Clío, 51-55.

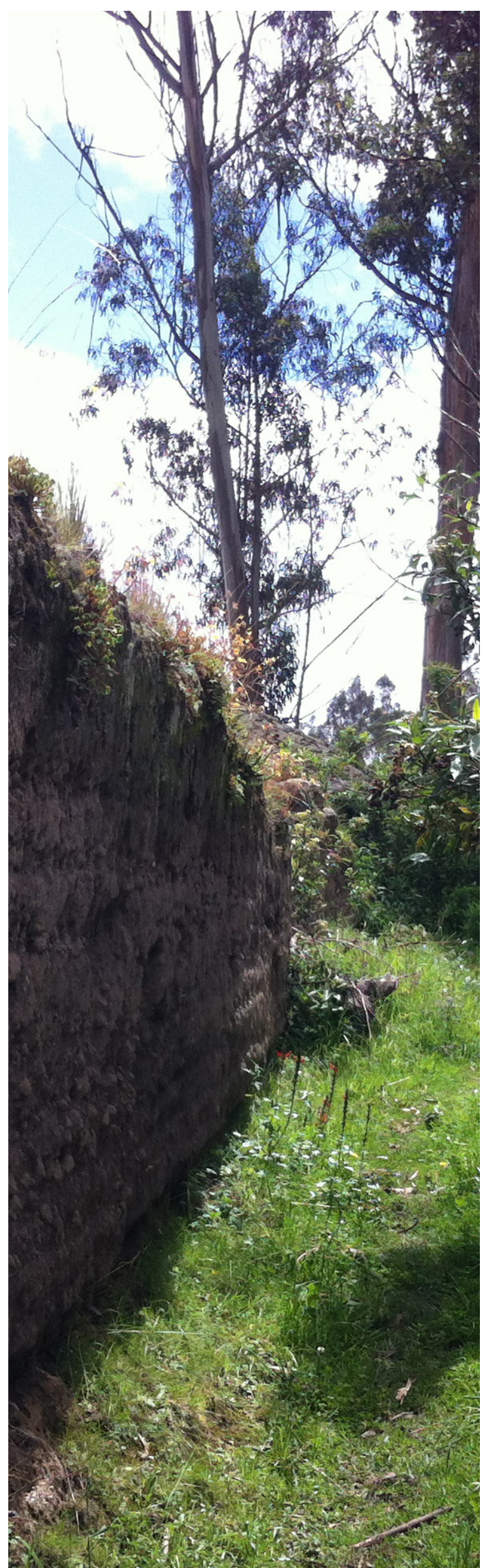

